\title{
Mathematical and computational tools in theoretical chemistry
}

\author{
J. Vigo-Aguiar • E. J. Brändas
}

Received: 14 September 2009 / Accepted: 15 September 2009 / Published online: 26 September 2009 (C) Springer Science+Business Media, LLC 2009

It is with pleasure that we offer the readers of the Journal of Mathematical Chemistry, JCM, this Special Issue consisting of the contributions to Mathematical and computational tools in theoretical chemistry presented at the 8th International Conference of Computational and Mathematical Methods in Science and Engineering, CMMSE2008, held at Hotel Meliá Galúa, La Manga del Mar Menor, Murcia, Spain, June 13-16, 2008. This special issue is another one in a line of CMMSE special issues, prior advances are in the references [1-5].

As the main emphasis and profile of each CMMSE conference state, viz. they are multi-disciplinary, they combine important and novel mathematical and computational methods as well as innovative modelling and algorithms common to a wide spectrum of areas of human activity and finally new insights and understanding of the necessary development and evolution of present state of the art of mathematical and computational techniques and models.

In this particular issue we will find, as the first article, important improvements in convergence and stability of applications to birth and death processes in chemical phenomena via iterative refinement of a certain class of matrices. A completely different contribution concerning neutron rich matter then follows. Since the methods, used here by the author, have a large overlap with the standard machinery of Quantum Chemistry, for instance the interaction model, the spherical harmonics analysis, and further with general techniques used in Theoretical Chemistry and Chemical Physics, e.g. cluster simulations, thermodynamic dependencies etc., the editors found the

\footnotetext{
J. Vigo-Aguiar ( $\varangle)$

Universidad de Salamanca, Salamanca, Spain

e-mail: jvigo@usal.es
}

\section{E. J. Brändas}

University of Uppsala, Uppsala, Sweden

e-mail: erkki@kvac.uu.se 
submission relevant for publication in this special volume of JCM. The next paper reports on a very simple method for finding a suitable initial estimation for dynamic optimization in chemical processes. Several examples, from unconstrained to constrained scenarios and finally to the very difficult no differentiable optimal control problem are given.

Recently there has been an increased interest in applications of information theory in theoretical chemistry bearing e.g. on the close relationship between kinetic energy functionals and Fisher information. In this contribution an interesting link between the Thomas Fermi method and the Shannon entropy is examined and its consequences analysed. In the two final reports non-standard, non-uniform mesh techniques are studied for solving general non-linear problems, providing third order convergence. Finally a powerful exponential fitting formula is applied to the time dependent Schrödinger equation. The authors conclude that the approach describe the second spatial derivative much better than standard finite difference approximation leading up to an increase of accuracy from fourth order to sixth order with only little more computational effort.

Nowadays the use of meshless methods is increasing in the numerical solution of partial differential equations and the applications of this technique in Chemistry are growing up. We have selected a last paper illustrating these techniques on modelling the electromagnetic behaviour of a single wall carbon nanotube.

All in all we find this special issue an informative and useful collection of papers and we hope that the readers of JCM will enjoy the reading as much as the editors did in putting them together. If the reader is interested in the presented techniques he can find interesting the above references.

Finally we want to thank Professor Paul G. Mezey and all the authors for their help in contributing excellently to this special volume. We are grateful for the support received to this issue from the I-Math project FUT-C2-0039. We also like to thank the JCYL project SA/050A08 and the Spanish CICYT MTM2008-05489.

\section{References}

1. J. Vigo-Aguiar, B.A. Wade, Recent advances in computational and applied mathematics in science and engineering. Int. J. Comput. Math. 85(3-4), (2008)

2. J. Vigo-Aguiar, B.A. Wade, Special issue on the international conference of computational methods in sciences and engineering (ICCMSE 2005)—Dedicated to Prof. Erkki J. Brandas. J. Math. Chem. 40(1), (2006)

3. J. Vigo-Aguiar, Special issue on computational and mathematical methods in science and engineering (CMMSE-2004). J. Comput. Appl. Math. 192(1), (2006)

4. T.E. Simos, J. Vigo-Aguiar, Special issue for the international conference of computational methods in sciences and engineering 2003 (ICCMSE 2003). Math. Comput. Model. 42(7-8), (2005)

5. T.E. Simos, J. Vigo-Aguiar, Special issue - selected papers from the conference on computational and mathematical methods for science and engineering (CMMSE-2002). J. Comput. Appl. Math. 158(1), (2003) 